\title{
RELATIONS BETWEEN LOOP IDENTITIES
}

R. ARTZY

I. It is well known $[3]^{1}$ that every loop $\mathfrak{Q}$ can be represented as a loop of a 3-net. Interchanges of the line families of the net may be used for the construction of new loops over the same set of elements as $\mathfrak{R}$, but with different operations. The requirement that the operation of one of these loops coincide with that of $\&$ leads to an identity in $\mathfrak{R}$, and the knowledge of the group of interchanges permits systematic conclusions as to the implicative relations between certain loop identities. In the past such relations were discovered $[1 ; 3 ; 4]$ only rather incidentally. The present method also yields results about the nuclei of loops with identities. The same procedure could be applied to double loops and 4-nets, in the sense of [5, p. 61], and certain rules of distributivity could be obtained and related to each other; however in the present note this subject will not be dealt with.

A similar method has been used by G. Bol. [2, pp. 419, 422] for finding relations between certain configurations, later called "Bol configurations," in a 3-net (cf. [5, pp. 53-56]).

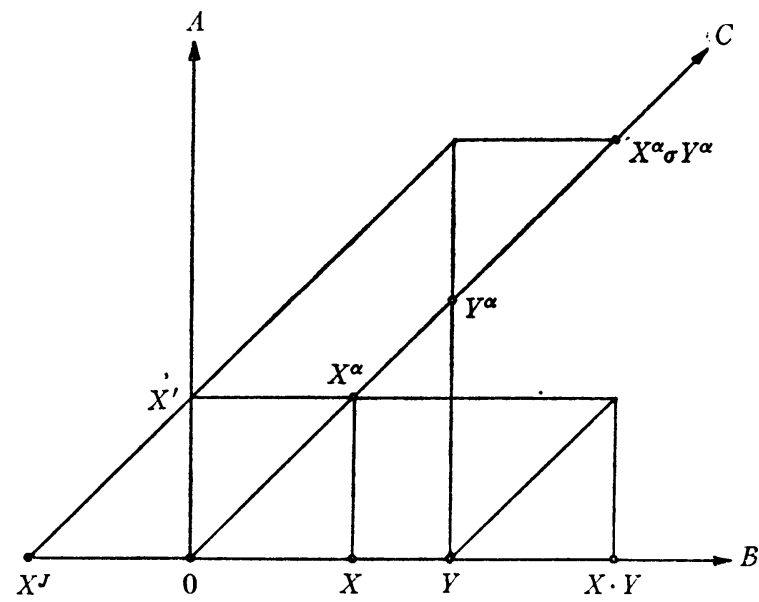

II. Let $\mathfrak{R}$ be an $(A, B, C)$-net (for the notation cf. [5, pp. 42-45]), $O$ a proper point of $\mathfrak{N}$, and $\mathfrak{R}=\mathfrak{R}(\mathfrak{N}, O)$, written multiplicatively, the corresponding loop. Accordingly we have for the proper points $X$

Presented to the Society, February 28, 1959; received by the editors August 1, 1959, and, in revised form, January 25, 1960.

${ }^{1}$ Wherever possible, reference is made to the comprehensive texts $[3 ; 5]$ rather than to the original papers. 
and $Y$ on $O B, X \cdot Y=((X A \cap O C) B \cap Y C) A \cap O B$. We define the biunique mapping $\alpha: X^{\alpha}=X A \cap O C$ of the elements of $\&$ upon points of $O C$. In particular, $O^{\alpha}=O$. Now consider the $(B, C, A)$-net $\mathfrak{N}^{\sigma}$ and the loop $\mathfrak{R}\left(\mathfrak{R}^{\sigma}, O\right)$ whose binary operation $(\sigma)$ over the $X^{\alpha}$, all $X$ in $\mathfrak{R}$, is defined in the same way as the original multiplication was introduced in $\&$, namely $X^{\alpha} \sigma Y^{\alpha}=\left(\left(X^{\alpha} B \cap O A\right) C \cap Y^{\alpha} A\right) B \cap O C$. If $X^{J}$ is defined by $X \cdot X^{J}=0$, we have now $\left(X^{\alpha} \sigma Y^{\alpha}\right)^{\alpha^{-1}} \cdot X^{J}=Y,\left(X^{\alpha} \sigma Y^{\alpha}\right)^{\alpha^{-1}}$ $=Y / X^{J}$, and $X^{\alpha} \sigma Y^{\alpha}=\left(Y / X^{J}\right)^{\alpha}$.

Similarly, in the $(A, C, B)$-net $\mathfrak{N}^{\tau}$, we have the loop $\mathfrak{R}\left(\mathfrak{R}^{\tau}, O\right)$ with the binary operation $(\tau)$ over the $X^{\alpha}$, all $X$ in $\mathfrak{R}(\mathfrak{R}, O)$. Here we get $X^{\alpha} \tau Y^{\alpha}=(Y \cdot X)^{\alpha}$.

Now, performing successively the interchanges $\sigma$ and $\tau$ in a prearranged order, we can define $\mathfrak{N}^{p}$, where $\rho$ is any element of the group generated by $\sigma$ and $\tau$ under composition.

THEOREM 1. The group (5) generated by $\sigma$ and $\tau$ has defining relations

$$
\tau^{2}=(\sigma \tau)^{2}=1
$$

Thus BS is the infinite dihedral group or one of its homomorphs.

Proof. Consider the loop $R^{2}=\left(\mathbb{R}^{r}\right)^{\tau}$. It belongs to an $(A, B, C)$-net because $\tau$ brings about a permutation

$$
\left(\begin{array}{l}
123 \\
132
\end{array}\right)
$$

of the net families, and this permutation has order 2 . Here the first $\alpha$ maps $X \in O B$ on $X A \cap O C=X^{\alpha}$, and the second $\alpha$ carries $X^{\alpha} \rightarrow X^{\alpha} A$ $\cap O B=X$. Hence $\tau^{2}=1$.

We deal now with $(\sigma \tau)^{2}$. The permutation belonging to $\sigma$ is

$$
\left(\begin{array}{l}
123 \\
312
\end{array}\right)
$$

and $(\sigma \tau)^{2}$ yields the product

$$
\left(\begin{array}{l}
123 \\
312
\end{array}\right)\left(\begin{array}{l}
123 \\
132
\end{array}\right)\left(\begin{array}{l}
123 \\
312
\end{array}\right)\left(\begin{array}{l}
123 \\
132
\end{array}\right)
$$

which is the identity permutation. The four mappings $\alpha$ in turn act as follows:

$$
X \rightarrow X^{\alpha}, \quad X^{\alpha} \rightarrow X^{\alpha} B \cap O A=X^{\prime}, \quad X^{\prime} \rightarrow X^{\alpha}, \quad X^{\alpha} \rightarrow X .
$$

Hence $(\sigma \tau)^{2}=1$. Having thus ascertained the role of the $\alpha$ 's we shall omit them from here on, writing, for instance, $X \tau Y=Y \cdot X$.

In view of $\tau^{2}=1, \tau^{-1}=\tau$, every additional relation of \&f can be 
written in the form $\tau^{{ }^{e 1}} \sigma^{e 1} \tau \sigma^{e 2} \tau \cdots \tau \sigma^{e k} \tau^{e_{2}}=1$, where the $e^{\text {'s }}$ are integers and the $\epsilon$ 's either 0 or 1 . From (1) we have $\sigma \tau=\tau \sigma^{-1}$ and $\sigma^{-1} \tau$ $=\tau \sigma$. Thus successively all the $\tau$ 's can be shifted to the left, and we obtain $\tau^{\epsilon} \sigma^{s}=1$. Now, any relation of $\&$ would have to be fulfilled also in the permutation group of the three net families, that is, the symmetric group $S_{3}$. In other terms, $S_{3}$ is a homomorph of (5). But $\tau^{\epsilon} \sigma^{e}=1$ is satisfied in $S_{3}$ only when $\epsilon=0, e=3 n, n$ an integer. The resulting relation $\sigma^{3 n}=1$ means $X \sigma^{3 n} Y=X \cdot Y$. We use $R$ and $L$, respectively, for right and left multiplication with subscripts denoting operations other than $(\cdot)$. Then $X \sigma Y=Y / X^{J}=Y R^{-1}\left(X^{J}\right)$, and by iteration, $X \sigma^{2} Y=Y R_{\sigma}^{-1}\left(X^{J}\right)=\left(X^{J} L^{-1}(Y)\right)^{J^{-1}}$. Hence $X \sigma^{3} Y$ $=\left(X^{J} L_{\sigma}^{-1}(Y)\right)^{J^{-1}}, Y \sigma\left(X \sigma^{3} Y\right)^{J}=X^{J}$, and $X \sigma^{3} Y=\left(X^{J} \cdot Y^{J}\right)^{J^{-1}}$. Repeating this further we obtain $X \sigma^{3 n} Y=\left(X^{J^{n}} \cdot Y^{J^{n}}\right)^{J^{-n}}$, with $n$ any positive integer. This result can be easily extended to negative integers $n$. Combined with $X \sigma^{3 n} Y=X \cdot Y$ from above, we have $(X \cdot Y)^{J^{n}}=X^{J^{n}}$ - $Y^{J^{n}}$. Loops satisfying this identity have been constructed for several values of $n$, and in these cases $B$ is a homomorph of the infinite dihedral group whose defining relations are (1). On the other hand, a free loop will not satisfy the identity for any $n$, and then $\mathbb{B}$, having no other defining relations besides (1), is the infinite dihedral group itself.

III. In this section the following, rather obvious, rules will be of importance.

(a) If $\rho_{1}, \cdots, \rho_{k}$ are elements of \&s and if $\rho=\rho_{1} \cdots \rho_{k}$, then the simultaneous validity of the identities $X \rho_{i} Y=X \cdot Y$, for $i=1, \cdots, k$, implies the identity $X \rho Y=X \cdot Y$, all $X, Y$ in $\&$.

(b) $X \rho Y=X \cdot Y$ implies $X \rho^{-1} Y=X \cdot Y$.

Applications. We now show that certain of the identities of the form $X \rho Y=X \cdot Y$ are equivalent to well-known loop identities.

(i) $X \sigma Y=Y R^{-1}\left(X^{J}\right)=X \cdot Y$ yields $Y=(X \cdot Y) \cdot X^{J}$, the crossedinverse property [3].

(ii) $X \sigma^{2} Y=\left(X^{J} L^{-1}(Y)\right)^{J^{-1}}=X \cdot Y$ yields $X^{J}=Y \cdot(X \cdot Y)^{J}$, the weak inverse property [4].

(iii) $X \sigma^{3} Y=\left(X^{J} \cdot Y^{J}\right)^{J^{-1}}=X \cdot Y$ yields $X^{J} \cdot Y^{J}=(X \cdot Y)^{J}$, the automorphic-inverse property [1].

(iv) $X \sigma \tau Y=X R^{-1}\left(Y^{J}\right)=X \cdot Y$ yields $X=(X \cdot Y) \cdot Y^{J}$, the right inverse-property [3].

(v) $X \tau \sigma Y=Y L^{-1}\left(X^{J^{-1}}\right)=X \cdot Y$ yields $Y=X^{J^{-1}} \cdot(X \cdot Y)$, the left inverse-property [3].

(vi) $X \tau Y=Y \cdot X=X \cdot Y$ yields commutativity.

(vii) $X \sigma^{3} \tau Y=\left(Y^{J} \cdot X^{J}\right)^{J^{-1}}=X \cdot Y$ yields $(X \cdot Y)^{J}=Y^{J} \cdot X^{J}$ of $[5$, p. 56]. 
(viii) $X \sigma^{3 n} Y=\left(X^{J^{n}} \cdot Y^{J^{n}}\right)^{J^{-n}}=X \cdot Y$ yields $X^{J^{n}} \cdot Y^{J^{n}}=(X \cdot Y)^{J^{n}}$.

From our rules (a), (b), information can be obtained concerning the implicative relations between these identities. A few examples follow.

The crossed-inverse property implies the weak inverse property because $\sigma^{2}=\sigma \cdot \sigma$, and the automorphic-inverse property because $\sigma^{3}=\sigma \cdot \sigma \cdot \sigma$.

The weak inverse property implies $X^{J^{2}} \cdot Y^{J^{2}}=(X \cdot Y)^{J^{2}}$ because $\sigma^{2} \cdot \sigma^{2} \cdot \sigma^{2}=\sigma^{6}$.

The right and left inverse-properties together imply $(X \cdot Y)^{J}$ $=Y^{J} \cdot X^{J}$ because $\sigma \tau \cdot \tau \sigma \cdot \sigma \tau=\sigma^{3} \tau$.

IV. Theorem 2. The left nucleus $N_{L}$, the middle nucleus $N_{M}$ and the right nucleus $N_{R}$ of a loop \& coincide, respectively, with the right, left and middle nuclei of $\mathfrak{R}^{\sigma}$; they are also, respectively, the right, middle and left nuclei of $\mathbf{R}^{\mathrm{T}}$.

PROOF. A more transparent proof would have to use geometrical arguments based upon the symmetry properties of the Reidemeister figure. This would explain the fact that the nuclei $N_{L}, N_{M}, N_{R}$ are shifted by elements of $(B)$ according to the same permutations as the net line families. But for practical reasons an algebraic proof will be given here.

In the following, loop elements will be denoted by lower case letters and the unit element by 1 .

(i) Let $x \in N_{L}$, then

$$
x a \cdot b=x \cdot a b \quad \text { for all } a, b \text { in } \& \text {. }
$$

Put $c=a b$, then $(x \cdot c / b) b=x c$, and

$$
x \cdot c / b=(x c) / b .
$$

Now, from (2) with $b=a^{J}, x a \cdot a^{J}=x, x a=x / a^{J}$. Applied to (3) this yields

$$
x /(c / b)^{J}=\left(x / c^{J}\right) / b
$$

or $\left(b^{J^{-1}} \sigma c\right) \sigma x=b^{J^{-1}} \sigma(c \sigma x)$.

Conversely, (4) with $b=c$ yields $x=\left(x / c^{J}\right) / c$ and $x c=x / c^{J}$. Applied to (4) this gives $x \cdot c / b=(x c) / b,(x \cdot c / b) b=x c$ and, with $c / b=a$, $x a \cdot b=x \cdot a b$.

(ii) Given $y \in N_{M}$, that is, $a y \cdot b=a \cdot y b$ for all $a, b$ in $\mathbb{R}$. Since a nucleus is a group $\left[3\right.$, p. 57], also $a y^{J} \cdot b=a \cdot y^{J} b$. Call $a y^{J} \cdot b=c$, then $c=\left((c / b) / y^{J}\right) \cdot y^{J} b$ and

$$
c /\left(y^{J} b\right)=(c / b) / y^{J} .
$$


With $c=1$ this becomes $\left(y^{J} b\right)^{J^{-1}}=b^{J^{-1}} / y^{J}$ and

$$
y^{J} b=\left(b^{J^{-1}} / y^{J}\right)^{J}
$$

Applied to (5) this yields

$$
c /\left(b^{J^{-1}} / y^{J}\right)^{J}=(c / b) / y^{J},
$$

that is, $\left(y \sigma b^{J^{-1}}\right) \sigma c=y \sigma\left(b^{J^{-1}} \sigma c\right)$.

Conversely, setting $c=y^{\mathrm{J}} b$ in (7) yields (6), which allows the argument to be reversed as in (i).

(iii) If $z \in N_{R}$, then also $a \cdot b z^{J}=a b \cdot z^{J}$ for all $a, b$ in $\&$. Put $a b \cdot z^{J}=c$, then $c=\left(c / z^{J}\right) / b \cdot b z^{J}, c /\left(b z^{J}\right)=\left(c / z^{J}\right) / b$. With $c=1$ this is $b z^{J}=(z / b)^{J}$, and, substituted back,

$$
c /(z / b)^{J}=\left(c / z^{J}\right) / b,
$$

that is, $\left(b^{J^{-1}} \sigma z\right) \sigma c=b^{J^{-1}} \sigma(z \sigma c)$.

Conversely, put $\left(c / z^{J}\right) / b=a$, then from (8), $\left(a b \cdot z^{J}\right) /(z / b)^{J}=a$, $a b \cdot z^{J}=a(z / b)^{J}$. With $a=1, b z^{J}=(z / b)^{J}$. Substituted back, $a b \cdot z^{J}$ $=a \cdot b z^{J}$.

(iv) The proof for the operation $(\tau)$ is trivial.

CoROLlaRIES. 1. In a weak inverse loop the three nuclei coincide [4], and so do the nuclei in a crossed-inverse loop [1].

Proof. Let $N^{\rho}$ be a nucleus of $\mathfrak{R}^{\rho}, \rho \in(\$)$. Then in the weak inverse loop $N_{L}=N_{M}^{\sigma^{2}}=N_{M}, N_{M}=N_{R}^{\sigma^{2}}=N_{R}$. The second statement is obvious since the weak inverse property holds in every crossed-inverse loop.

2. In a left I.P. loop the left and middle nuclei coincide.

Proof. $N_{L}=N_{M}^{\tau \sigma}=N_{M}$.

3. In a right I.P. loop the right and middle nuclei coincide.

4. In loops with the property $(x y)^{J}=y^{J} x^{J}$ the right and left nuclei coincide.

Proof. $N_{L}=N_{R}^{\sigma^{3} \tau}=N_{R}$.

\section{REFERENCES}

1. R. Artzy, Crossed-inverse and related loops, Trans. Amer. Math. Soc. vol. 91 (1959) pp. 480-492.

2. G. Bol, Gewebe und Gruppen, Math. Ann. vol. 114 (1937) pp. 414-431. 1958.

3. R. H. Bruck, $A$ survey of binary systems, Berlin-Göttingen-Heidelberg, Springer,

4. J. M. Osborn, Loops with the weak inverse property, Pacific J. Math. vol. 10 (1960) pp. 295-304.

5. G. Pickert, Projektive Ebenen, Berlin-Göttingen-Heidelberg, Springer, 1955.

ISRAEL INSTITUTE OF TEChNOLOGY 